\title{
Pretreatment neutrophil-to-lymphocyte ratio predicts the prognosis in patients with metastatic prostate cancer
}

Takashi Kawahara ${ }^{1,2^{*}+} \mathbb{D}$, Yumiko Yokomizo ${ }^{1 \dagger}$, Yusuke Ito ${ }^{1}$, Hiroki Ito ${ }^{1}$, Hitoshi Ishiguro ${ }^{3}$, Jun-ichi Teranishi ${ }^{2}$, Kazuhide Makiyama ${ }^{1}$, Yasuhide Miyoshi ${ }^{2}$, Hiroshi Miyamoto ${ }^{4}$, Masahiro Yao ${ }^{1}$ and Hiroji Uemura ${ }^{2}$

\begin{abstract}
Background: The neutrophil-to-lymphocyte ratio (NLR), a simple marker of the systemic inflammatory response in critical care patients, has been suggested as an independent prognostic factor for several solid malignancies. We investigated the utility of pretreatment NLR as a prognosticator in patients who presented with metastatic prostate cancer.

Methods: We first investigated the correlation between NLR and prostate-specific antigen (PSA) levels in 1464 men who had both tests and were found to have prostate cancer on their biopsies at our institution from 1999 to 2015. We then assessed the relationship between pretreatment NLR and the prognosis in 48 patients who were diagnosed with prostate cancer metastasized to the lymph node and/or bone.

Results: The NLR value was significantly elevated in men with higher PSA than in those with lower PSA $(p<0.001)$. In patients with metastatic prostate cancer, NLR (cut-off point of 3.37 determined by the AUROC curve) was correlated with both cancer-specific $(p=0.018)$ and overall $(p=0.008)$ survivals.
\end{abstract}

Conclusions: Pretreatment NLR may function as a new biomarker that precisely predicts the prognosis in patients with metastatic prostate cancer.

Keywords: Prostate cancer, Biomarker, Neutrophil-to-lymphocyte ratio, Metastasis

\section{Background}

Prostate cancer is the most common malignancy in men. The PSA screening test is widely available in Japan, but a large number of patients still present with advanced stage prostate cancer. Although most of prostate cancers with metastatic lesions respond to initial androgen ablation therapy, responders ultimately develop progressive disease of hormone refractory cancer.

The neutrophil-to-lymphocyte ratio (NLR) has been suggested as a simple marker of the systemic inflammatory response in critical care patients [1]. It has also been reported as an independent prognostic factor for several

\footnotetext{
* Correspondence: takashi_tk2001@yahoo.co.jp

${ }^{\dagger}$ Equal contributors

${ }^{1}$ Department of Urology, Yokohama City University, Graduate School of Medicine, Yokohama, Japan

²Departments of Urology and Renal Transplantation, Yokohama City Medical

Center, Yokohama, Japan

Full list of author information is available at the end of the article
}

solid malignancies [2-11]. Importantly, NLR can easily be calculated from routine complete blood counts (CBCs) in peripheral blood samples $[9,10]$.

The utility of NLR as a potential biomarker for prostate cancer has been investigated [12]. However, most of these have included patients with advanced tumor, such as castration-resistant prostate cancer, or those who received second-line chemotherapy. To our knowledge, no studies have assessed pretreatment NLR as a predictive marker of survival in patients who were diagnosed with metastatic prostate cancer.

\section{Methods \\ Patients and clinical and laboratory assessments \\ A total of 73,637 CBCs examinations that included absolute neutrophil and lymphocyte counts were performed in 9782 men at the Department of Urology, Yokohama City University Hospital (Yokohama, Japan) from 1999 to}


2015. Both CBCs and PSA levels were examined in 1464 patients who were diagnosed with prostate cancer, including 48 presented with prostate cancer metastasized to the lymph node and/or bone. As the initial treatment, 42 $(87.5 \%)$ received combine androgen blockade, 8 (18.8 \%) received zoledronic acid, 3 (7.1 \%) underwent radical prostatectomy, and none of the patients received radiation therapy. All patients had no systemic inflammation at the time of biopsy. This study was approved by the Institutional Review Board of the Yokohama City University Medical Center. Written informed consent was obtained from all patients.

\section{Statistical analysis}

The patients' characteristics were analyzed using the Mann-Whitney $U$, chi-square, and one factor ANOVA tests. Any correlations between the variables were determined by the Spearman correlation analysis. The NLR cut-off value was evaluated by the AUROC curve. The Kaplan-Meier product limit estimator was used to estimate cancer-specific survival (CSS) and overall survival (OS). Survival duration was defined as the time between the dates of pathological diagnosis and death. A log-rank test was performed for comparison. The statistical analyses were performed using the Graph Pad Prism software program (Graph Pad Software, La Jolla, CA, USA). Statistical significance was determined as $p<0.05$.

\section{Results}

\section{NLR is positively associated with PSA}

A total of 1464 patients with prostate cancer were analyzed in this study. Their background information, including age, white blood cell count $(/ \mathrm{mL})$, neutrophil (\%), lymphocyte (\%), and NLR are described in Tables 1 and 2 . In patients with PSA of $<4 \mathrm{ng} / \mathrm{mL}$, the mean NLR was 2.57 , which was increased up to 6.43 in those with PSA of $\geq 500 \mathrm{ng} / \mathrm{mL}$ (Fig. 1). Therefore, the NLR values were significantly higher in the high PSA level groups $(p<0.001)$. On the other hand, because of the exponential increases in the PSA levels, the correlation coefficient was not significantly different $(r=0.09, p=0.08)$.

\section{NLR predicts the survival of metastatic prostate cancer}

CBCs were examined in 48 metastatic prostate cancer patients before undergoing prostate needle biopsy. The basic characteristics, including age, initial PSA, and Gleason score, are shown in Table 3. There were no statistically significant correlations between NLR and each of clinicopathologic feature. The median and mean $( \pm$ SD) followup times were 61 and $55.9( \pm 34.0)$ months, respectively. Nineteen patients (39.6\%) died of prostate cancer.

We created an AUROC curve to determine the NLR cut-off value for predicting the prognosis. The cut-off value was determined to be 3.37 (Fig. 2). Patients with
Table 1 Characteristics of 1464 patients with prostate cancer

\begin{tabular}{|c|c|}
\hline Variables & $n(\%)$, or median (range, mean \pm SD) \\
\hline \multicolumn{2}{|l|}{ PSA $<4$} \\
\hline Number of patients & $738(50.4 \%)$ \\
\hline Age (y) & $76(35-99,75.3 \pm 9.0)$ \\
\hline $\mathrm{WBC}(/ \mathrm{mL})$ & $5800(2000-16,100,6321.3 \pm 2203.3)$ \\
\hline Neutrophil (\%) & $58.8(4.5-89.0,59.5 \pm 10.3)$ \\
\hline Lymphocyte (\%) & $29.0(1.0-60.5,28.6 \pm 8.9)$ \\
\hline NLR & $2.02(0.09-44.5,2.57 \pm 2.33)$ \\
\hline \multicolumn{2}{|l|}{$4 \leq$ PSA $<20$} \\
\hline Number of patients & $519(35.5 \%)$ \\
\hline Age (y) & $73(44-99,72.8 \pm 8.2)$ \\
\hline $\mathrm{WBC}(/ \mathrm{mL})$ & $5900(1100-22,800,6286.1 \pm 2056.8)$ \\
\hline Neutrophil (\%) & $60.5(3.0-90.0,60.6 \pm 10.4)$ \\
\hline Lymphocyte (\%) & $28.6(3.0-91.0,28.6 \pm 9.2)$ \\
\hline NLR & $2.11(0.03-28.67,2.57 \pm 1.99)$ \\
\hline \multicolumn{2}{|l|}{$20 \leq P S A<100$} \\
\hline Number of patients & $113(7.7 \%)$ \\
\hline Age (y) & $76(54-104,76.2 \pm 8.9)$ \\
\hline $\mathrm{WBC}(/ \mathrm{mL})$ & $6000(1400-28,500,6379.7 \pm 3919.7)$ \\
\hline Neutrophil (\%) & $61.4(7.0-96.7,61.6 \pm 13.1)$ \\
\hline Lymphocyte (\%) & $27.7(2.6-82.5,26.9 \pm 11.9)$ \\
\hline NLR & $2.21(0.08-37.19,3.64 \pm 4.78)$ \\
\hline \multicolumn{2}{|l|}{$100 \leq P S A<500$} \\
\hline Number of patients & $53(3.6 \%)$ \\
\hline Age (y) & $77(59-99,78.0 \pm 8.6)$ \\
\hline $\mathrm{WBC}(/ \mathrm{mL})$ & $6300(1000-26,400,6664.2 \pm 3501.1)$ \\
\hline Neutrophil (\%) & $66.0(25.5-95,64.1 \pm 15.1)$ \\
\hline Lymphocyte (\%) & $22.5(2.5-61.0,23.3 \pm 11.9)$ \\
\hline NLR & $2.83(0.42-38.00,4.60 \pm 5.75)$ \\
\hline \multicolumn{2}{|l|}{$500 \leq$ PSA } \\
\hline Number of patients & $41(2.8 \%)$ \\
\hline Age (y) & $79(60-95,77.3 \pm 9.1)$ \\
\hline $\mathrm{WBC}(/ \mathrm{mL})$ & $6400(2300-48,800,7973.2 \pm 6909.6)$ \\
\hline Neutrophil (\%) & $69.0(29.0-60.0,67.0 \pm 14.8)$ \\
\hline Lymphocyte (\%) & $20.0(1.5-58.0,22.2 \pm 13.0)$ \\
\hline NLR & $3.33(0.50-60.00,6.43 \pm 10.49)$ \\
\hline
\end{tabular}

high NLR showed significantly poorer CSS $(p=0.018)$ and OS $(p=0.008)$, compared with those with low NLR (Fig. 3).

\section{Discussion}

PSA was also found to be positively correlated with the NLR in patients with prostate cancer. McDonald et al. hypothesized that the NLR might reflect the balance between innate (neutrophils) and adaptive (lymphocytes) immune responses; its association with higher serum 
Table 2 Patients' background

\begin{tabular}{|c|c|c|c|c|}
\hline Variables & Total & Low NLR $(<3.37, n=36)$ & High NLR $(\geq 3.37, n=12)$ & $p$ value \\
\hline Age $(y r)$ & $70.5(71.33 \pm 6.92)$ & $70(70.7 \pm 7.2)$ & $73.5(73.3 \pm 5.9)$ & 0.234 \\
\hline iPSA (ng/mL) & $82.2(605.1 \pm 1446.3)$ & $82.2(436.2 \pm 1090.7)$ & $102.1(1111.7 \pm 2186.0)$ & 0.322 \\
\hline \multicolumn{5}{|l|}{ Pathological Grade } \\
\hline Gleason's Sum $\leq 6$ & $2(4.2 \%)$ & $1(2.8 \%)$ & $1(8.3 \%)$ & \multirow[t]{3}{*}{0.156} \\
\hline Gleason's Sum = 7 & $14(29.2 \%)$ & $13(36.1 \%)$ & $1(8.3 \%)$ & \\
\hline Gleason's Sum $\geq 8$ & $32(66.7 \%)$ & $22(61.1 \%)$ & $10(83.3 \%)$ & \\
\hline \multicolumn{5}{|l|}{ Clinical T Stage } \\
\hline 2 & $6(12.5 \%)$ & $4(11.1 \%)$ & $2(16.7 \%)$ & \multirow[t]{3}{*}{0.501} \\
\hline 3 & $27(56.3 \%)$ & $22(61.1 \%)$ & $5(41.7 \%)$ & \\
\hline 4 & $15(31.3 \%)$ & $10(27.8 \%)$ & $5(41.7 \%)$ & \\
\hline \multicolumn{5}{|l|}{ Clinical N Stage } \\
\hline 0 & $21(43.8 \%)$ & $16(44.4 \%)$ & 5 (41.7 \%) & \multirow[t]{2}{*}{0.867} \\
\hline 1 & $27(56.3 \%)$ & $20(55.6 \%)$ & 7 (58.3 \%) & \\
\hline \multicolumn{5}{|l|}{ Clinical M Stage } \\
\hline 0 & $13(27.1 \%)$ & $12(33.3 \%)$ & $1(8.3 \%)$ & \multirow[t]{2}{*}{0.091} \\
\hline 1 & 35 (72.9 \%) & $24(66.7 \%)$ & $11(91.7 \%)$ & \\
\hline NLR & $2.49(2.93 \pm 1.66)$ & $2.17(2.25 \pm 0.64)$ & $4.26(4.98 \pm 2.08)$ & $<0.001$ \\
\hline
\end{tabular}

median (mean \pm SD)

PSA levels might indicate impairment in the adaptive host's ability to control inflammation [12]. The PSA level was shown to be a prognostic factor in prostate cancer patients with a PSA of $>500 \mathrm{ng} / \mathrm{mL}$, and higher PSA levels ( $>1000$ or $5000 \mathrm{ng} / \mathrm{mL}$ ) were associated with a poorer outcome [13]. Our results also support those data.

Our results showed pretreatment NLR to be a predictor of survival in patients presenting with metastatic

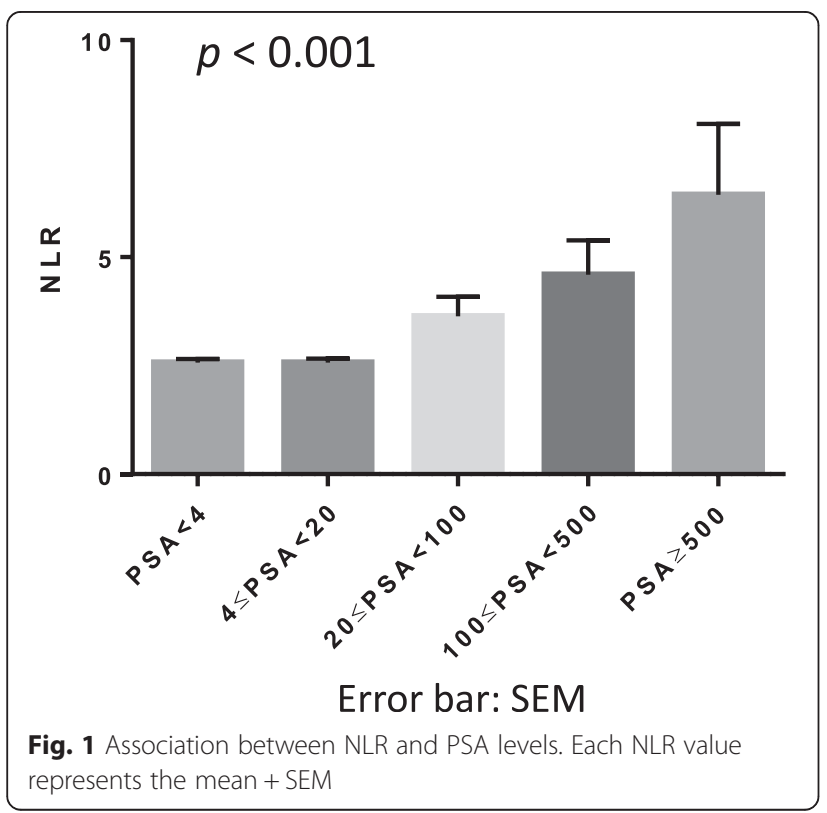

prostate cancer. Increasing evidence shows that the presence of systemic inflammation is correlated with poorer CSS in various solid organ tumors [3, 14-19]. Moreover, nonsteroidal anti-inflammatory medications have been suggested to reduce the risk of developing prostate cancer, implying its critical correlation with

Table 3 Characteristics of 48 patients with metastatic prostate cancer

\begin{tabular}{ll}
\hline Variables & Median (range, mean \pm SD) \\
\hline Age $(\mathrm{y})$ & $70.5(51-88,71.33 \pm 6.92)$ \\
Initial PSA (ng/mL) & $82.16(8.2-7285,605.06 \pm 1446.25)$ \\
Pathological Grade & \\
Gleason Score $\leq 6$ & $2(4.2 \%)$ \\
Gleason Score $=7$ & $14(29.2 \%)$ \\
Gleason Score $\geq 8$ & $32(66.7 \%)$ \\
Clinical T Stage & \\
2 & $6(12.5 \%)$ \\
3 & $27(56.3 \%)$ \\
4 & $15(31.3 \%)$ \\
Clinical N Stage & \\
0 & $21(43.8 \%)$ \\
1 & $27(56.3 \%)$ \\
Clinical M Stage & \\
0 & $13(27.1 \%)$ \\
1 & $35(72.9 \%)$ \\
NLR & $2.49(0.84-10.56,2.93 \pm 1.66)$ \\
\hline
\end{tabular}




\section{A Cancer-Specific Survival B Overall Survival}
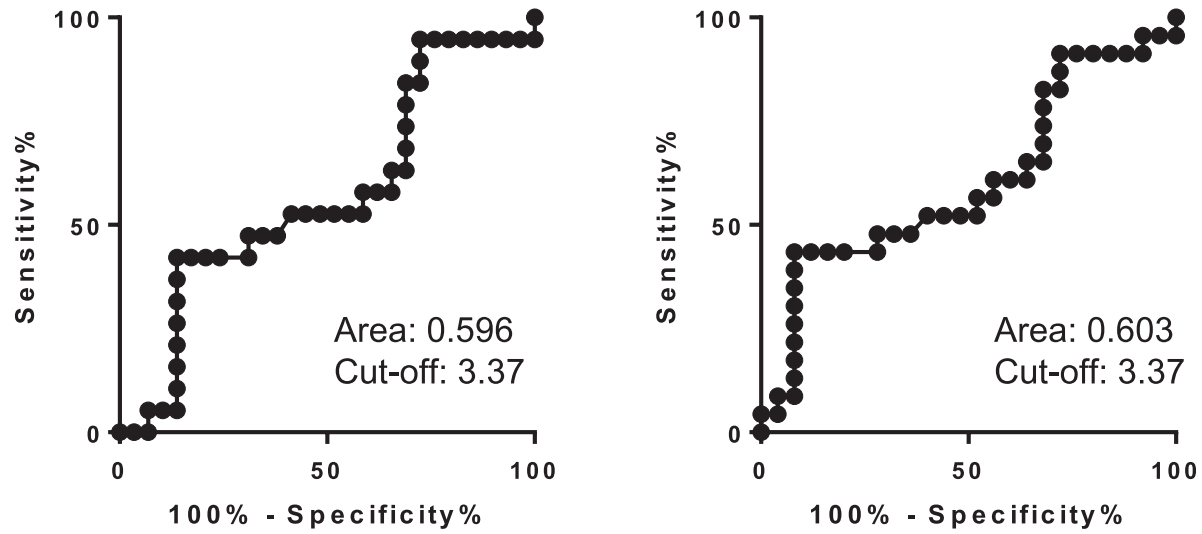

Fig. 2 The AUROC for the NLR (a: Cancer-Specific Survival, b: Overall Survival)

inflammation [14, 15]. Markers of systemic inflammation, including the NLR, have also been associated with elevated PSA levels in men without known prostatic disease [12]. It has previously been demonstrated that the presence of an inflammatory response can be determined by not only C-reactive protein expression but also NLR elevation $[1,3,20]$.

We retrospectively investigated the NLR in our clinical database. CBCs are usually determined during clinical check-ups, and thus it is possible to apply the NLR to all patients, both preoperatively and postoperatively. The NLR can be simply calculated from routine CBCs with differentials [21]. CBCs are usually examined in the clinical check-up, and NLR can be applied to virtually all patients either before or after surgery/ medical treatment. The NLR that has been proposed to estimate the magnitude of systemic inflammation in cancer patients is thus an easily and inexpensively measured prognostic marker [2, 22-24].
The proposed mechanisms involving the relationship between NLR elevation and tumor progression include the increased supply of growth factors, survival factors, pro-tumorigenic factors, and extracellular matrixmodifying enzymes (which can facilitate invasion and metastasis), and inductive signals that may lead to epithelial-to-mesenchymal transition [21, 25]. Patients with an elevated NLR have also been shown to exhibit a lymphocyte-mediated immune response to malignancy, suggesting that NLR elevation could be associated with an increased potential for tumor progression and a worse prognosis [26, 27]. The interaction between the tumor and the host immune system promotes tumor cell proliferation and metastasis, and activates the inflammatory cascade in the host, which leads to the further deterioration of the general condition of cancer patients [28]. Studies have proposed that tumor-associated neutrophils have two different states: anti-tumorigenic (N1-phenotype) and a pro-tumorigenic (N2-phenotype) [29, 30].
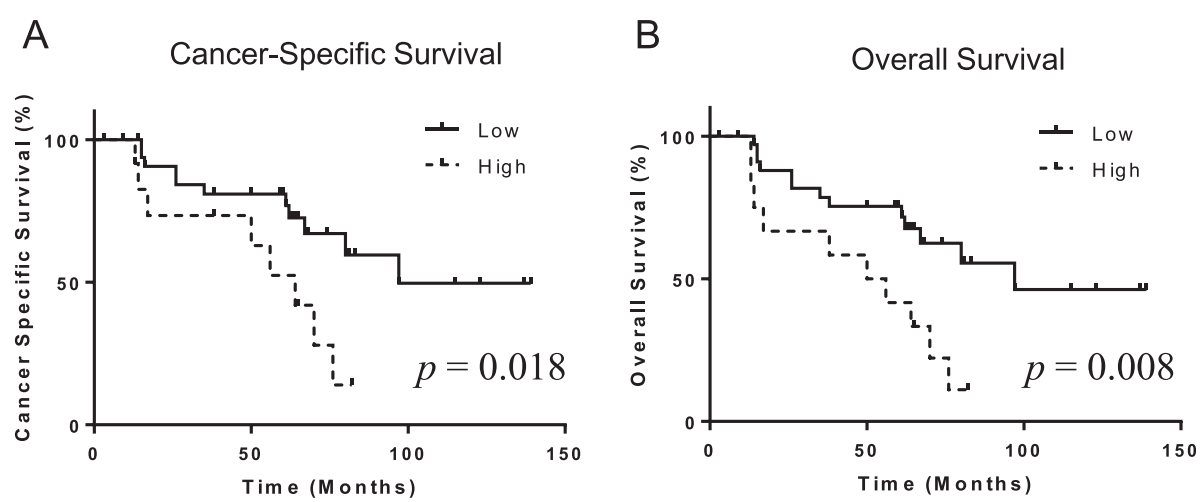

Fig. 3 The Association of patient outcomes with NLR (a: Cancer-Specific Survival, b: Overall Survival) 
CD8-positive lymphocytes are also known to play an antitumorigenic role [29, 31].

The present study is associated with some limitations due to its retrospective nature. Our patients received a variety of prostate cancer therapies including traditional hormonal treatment as well as several new drugs. Although the treatment options were heterogeneous, we found the NLR to be a new biomarker that was associated with patient outcomes. Thus, pretreatment NLR may be a useful prognosticator in patients with metastatic prostate cancer. The second one is that NLR may be affected by infection, systemic inflammation and medication, however, such conditions may not have affected the NLR in our cohort since the NLR was obtained at the time of biopsy and any cases with infection or systemic inflammation were excluded from the analysis. In addition, none of the patients received any prebiopsy medication that is known to affect the NLR. The third limitation is the limited sample size. As a result, further study with a larger group of patients is needed.

\section{Conclusions}

In conclusion, pretreatment NLR may function as a new biomarker that precisely predicts the prognosis in patients with metastatic prostate cancer.

\section{Availability of supporting data}

Due to ethical restrictions, the raw data underlying this paper is available upon request to the corresponding author.

\section{Abbreviations \\ CBCs: complete blood counts; CSS: cancer-specific survival; NLR: neutrophil-to-lymphocyte ratio; OS: overall survival; PSA: prostate-specific antigen.}

\section{Competing interests}

The authors declare that they have no competing interests.

\section{Authors' contributions}

Conceived and designed the experiments: TK, YY, HM, HU. Performed the experiments: YI, HIs, JT, KM, YM. Wrote the paper: TK, HIt, HM, MY. All authors have read and approved the manuscript.

\section{Acknowledgments}

Grants from the Uehara Memorial Foundation, the Tokyo Biochemical Research Foundation, the Japanese Foundation for Research and Promotion of Endoscopy, and an International Exchange Grant from Kato Memorial Bioscience Foundation were provided to T.K. There are no applicable grant numbers.

\begin{abstract}
Author details
${ }^{1}$ Department of Urology, Yokohama City University, Graduate School of Medicine, Yokohama, Japan. ${ }^{2}$ Departments of Urology and Renal Transplantation, Yokohama City Medical Center, Yokohama, Japan. ${ }^{3}$ Photocatalyst Group, Special Research Laboratory, Kanagawa Academy of Science and Technology, Kawasaki, Japan. ${ }^{4}$ Departments of Pathology and Urology, Johns Hopkins University School of Medicine, Baltimore, USA.
\end{abstract}

Received: 18 October 2015 Accepted: 7 February 2016

Published online: 16 February 2016

\section{References}

1. Zahorec R. Ratio of neutrophil to lymphocyte counts-rapid and simple parameter of systemic inflammation and stress in critically ill. Bratisl Lek Listy. 2001;102(1):5-14.

2. Xue P, Kanai M, Mori Y, Nishimura T, Uza N, Kodama Y, et al. Neutrophil-tolymphocyte ratio for predicting palliative chemotherapy outcomes in advanced pancreatic cancer patients. Cancer Med. 2014;3(2):406-15.

3. Gomez D, Morris-Stiff G, Toogood GJ, Lodge JP, Prasad KR. Impact of systemic inflammation on outcome following resection for intrahepatic cholangiocarcinoma. J Surg Oncol. 2008;97(6):513-8.

4. Chua W, Charles KA, Baracos VE, Clarke SJ. Neutrophil/lymphocyte ratio predicts chemotherapy outcomes in patients with advanced colorectal cancer. Br J Cancer. 2011:104(8):1288-95.

5. Azab B, Bhatt VR, Phookan J, Murukutla S, Kohn N, Terjanian T, et al. Usefulness of the neutrophil-to-lymphocyte ratio in predicting short- and long-term mortality in breast cancer patients. Ann Surg Oncol. 2012;19(1): 217-24

6. Dalpiaz O, Pichler M, Mannweiler S, Martin Hernandez JM, Stojakovic T, Pummer $\mathrm{K}$, et al. Validation of the pretreatment derived neutrophillymphocyte ratio as a prognostic factor in a European cohort of patients with upper tract urothelial carcinoma. Br J Cancer. 2014;110(10):2531-6.

7. Jung MR, Park YK, Jeong O, Seon JW, Ryu SY, Kim DY, et al. Elevated preoperative neutrophil to lymphocyte ratio predicts poor survival following resection in late stage gastric cancer. J Surg Oncol. 2011;104(5):504-10.

8. Demirtas A, Sabur V, Akinsal EC, Demirci D, Ekmekcioglu O, Gulmez I, et al. Can neutrophil-lymphocyte ratio and lymph node density be used as prognostic factors in patients undergoing radical cystectomy? Sci World J. 2013:2013:703579

9. Walsh SR, Cook EJ, Goulder F, Justin TA, Keeling NJ. Neutrophil-lymphocyte ratio as a prognostic factor in colorectal cancer. J Surg Oncol. 2005;91(3):181-4.

10. Ohno Y, Nakashima J, Ohori M, Hatano T, Tachibana M. Pretreatment neutrophil-to-lymphocyte ratio as an independent predictor of recurrence in patients with nonmetastatic renal cell carcinoma. J Urol. 2010;184(3):873-8.

11. Rosenberg L, Lawlor GO, Zenlea T, Goldsmith JD, Gifford A, Falchuk KR, et al Predictors of endoscopic inflammation in patients with ulcerative colitis in clinical remission. Inflamm Bowel Dis. 2013;19(4):779-84.

12. McDonald AC, Vira MA, Vidal AC, Gan W, Freedland SJ, Taioli E. Association between systemic inflammatory markers and serum prostate-specific antigen in men without prostatic disease - the 2001-2008 National Health and Nutrition Examination Survey. Prostate. 2014;74(5):561-7.

13. Sugihara T, Yu C, Kattan MW, Yasunaga H, Ihara H, Onozawa M, et al. Long-term survival of extremely advanced prostate cancer patients diagnosed with prostate-specific antigen over 500 ng/ml. Jpn J Clin Oncol. 2014;44(12):1227-32.

14. Kawahara $T$, Ishiguro $H$, Hoshino $K$, Teranishi J, Miyoshi $Y$, Kubota $Y$, et al. Analysis of NSAID-activated gene 1 expression in prostate cancer. Urol Int. 2010;84(2):198-202.

15. Ishiguro $\mathrm{H}$, Kawahara $\mathrm{T}$. Nonsteroidal anti-inflammatory drugs and prostatic diseases. BioMed Res Int. 2014;2014:436123.

16. Coussens LM, Werb Z. Inflammation and cancer. Nature. 2002;420(6917): 860-7.

17. Gunter MJ, Stolzenberg-Solomon R, Cross AJ, Leitzmann MF, Weinstein S, Wood RJ, et al. A prospective study of serum C-reactive protein and colorectal cancer risk in men. Cancer Res. 2006;66(4):2483-7.

18. Zhang K, Kaufman RJ. From endoplasmic-reticulum stress to the inflammatory response. Nature. 2008:454(7203):455-62.

19. Kawahara T, Miyoshi Y, Sekiguchi Z, Sano F, Hayashi N, Teranishi J, et al. Risk factors for metastatic castration-resistant prostate cancer (CRPC) predict long-term treatment with docetaxel. PLoS One. 2012;7(10):e48186.

20. McMillan DC, Canna K, McArdle CS. Systemic inflammatory response predicts survival following curative resection of colorectal cancer. Br J Surg. 2003:90(2):215-9.

21. Hermanns T, Bhindi B, Wei Y, Yu J, Noon AP, Richard PO, et al. Pre-treatment neutrophil-to-lymphocyte ratio as predictor of adverse outcomes in patients undergoing radical cystectomy for urothelial carcinoma of the bladder. Br J Cancer. 2014;111(3):444-51.

22. Stotz M, Gerger A, Eisner F, Szkandera J, Loibner H, Ress AL, et al. Increased neutrophil-lymphocyte ratio is a poor prognostic factor in patients with primary operable and inoperable pancreatic cancer. Br J Cancer. 2013;109(2): 416-21.

23. Smith RA, Bosonnet L, Raraty M, Sutton R, Neoptolemos JP, Campbell F, et al. Preoperative platelet-lymphocyte ratio is an independent significant 
prognostic marker in resected pancreatic ductal adenocarcinoma. Am J Surg. 2009;197(4):466-72.

24. Proctor MJ, Morrison DS, Talwar D, Balmer SM, O'Reilly DS, Foulis AK, et al. An inflammation-based prognostic score (mGPS) predicts cancer survival independent of tumour site: a Glasgow Inflammation Outcome Study. Br J Cancer. 2011;104(4):726-34.

25. Hanahan D, Weinberg RA. Hallmarks of cancer: the next generation. Cell. 2011;144(5):646-74.

26. Gondo T, Nakashima J, Ohno Y, Choichiro O, Horiguchi Y, Namiki K, et al. Prognostic value of neutrophil-to-lymphocyte ratio and establishment of novel preoperative risk stratification model in bladder cancer patients treated with radical cystectomy. Urology. 2012;79(5):1085-91.

27. Cho H, Hur HW, Kim SW, Kim SH, Kim JH, Kim YT, et al. Pre-treatment neutrophi to lymphocyte ratio is elevated in epithelial ovarian cancer and predicts survival after treatment. Cancer Immunol Immunother. 2009:58(1):15-23.

28. Mantovani A, Allavena P, Sica A, Balkwill F. Cancer-related inflammation. Nature. 2008:454(7203):436-44.

29. Wang J, Jia Y, Wang N, Zhang X, Tan B, Zhang G, et al. The clinical significance of tumor-infiltrating neutrophils and neutrophil-to-CD8+ lymphocyte ratio in patients with resectable esophageal squamous cell carcinoma. J Transl Med. 2014;12:7.

30. Fridlender ZG, Sun J, Kim S, Kapoor V, Cheng G, Ling L, et al. Polarization of tumor-associated neutrophil phenotype by TGF-beta: "N1" versus "N2" TAN. Cancer Cell. 2009;16(3):183-94.

31. Williams MA, Bevan MJ. Effector and memory CTL differentiation. Annu Rev Immunol. 2007;25:171-92.

\section{Submit your next manuscript to BioMed Central} and we will help you at every step:

- We accept pre-submission inquiries

- Our selector tool helps you to find the most relevant journal

- We provide round the clock customer support

- Convenient online submission

- Thorough peer review

- Inclusion in PubMed and all major indexing services

- Maximum visibility for your research

Submit your manuscript at www.biomedcentral.com/submit 